\title{
CHARACTERIZATION OF THE VARIABLE EXPONENT SOBOLEV NORM WITHOUT DERIVATIVES
}

\author{
PETER HÄSTÖ AND ANA MARGARIDA RIBEIRO*
}

\begin{abstract}
The norm in classical Sobolev spaces can be expressed as a difference quotient. This expression can be used to generalize the space to the fractional smoothness case. Since the difference quotient is based on shifting the function, it cannot be generalized to the variable exponent case. In its place, we introduce a smoothed difference quotient and show that it can be used to characterize the variable exponent Sobolev space.
\end{abstract}

\section{INTRODUCTION}

While studying the limit behavior of the Gagliardo semi-norms

$$
\|f\|_{W^{s, p}}^{p}=\int_{\Omega} \int_{\Omega} \frac{|f(x)-f(y)|^{p}}{|x-y|^{n+s p}} d x d y, \quad 0<s<1
$$

as $s \rightarrow 1$, Bourgain, Brézis, and Mironescu [3, 4] established the appropriate scaling factor for comparing the limit with the $L^{p}$-norm of the gradient of $f$. They characterize the Sobolev space $W^{1, p}$ and prove the convergence of certain imaging models of Aubert and Kornprobst [2] to the well-known total variation model of Rudin, Osher, and Fatemi $[15]$.

In variable exponent Sobolev spaces one cannot expect to have a similar characterization. Indeed, by $\left[9\right.$, Proposition 3.6.1] the translation operator is bounded on $L^{p(\cdot)}\left(\mathbb{R}^{n}\right)$ if and only if $p$ is constant. In particular, this means that in the difference quotient we cannot replace the exponent by $p(x)$ or $p(y)$. This problem was first encountered by Fiorenza [11] who proposed instead to use the exponent $p(x, y):=\min _{z \in[x, y]} p(z)$ which depends on two variables. Unfortunately, with this exponent the connection to the framework of $L^{p(\cdot)}$ spaces is not so clear. In [8], Diening and Hästö replaced the difference quotient in the definition of the trace space by a sharp averaging operator $M_{B(x, r)}^{\#}$. This is the approach followed also here that will lead to a characterization of variable exponent Sobolev spaces $W^{1, p(\cdot)}\left(\mathbb{R}^{n}\right)$.

Throughout the paper, $\left(\psi_{\varepsilon}\right)_{\varepsilon}$ denotes a family of functions such that

$$
\psi_{\varepsilon} \in L^{1}(0,1), \quad \psi_{\varepsilon} \geqslant 0, \quad \int_{0}^{1} \psi_{\varepsilon}(r) d r=1
$$

and for every $\gamma>0$

$$
\lim _{\varepsilon \rightarrow 0^{+}} \int_{\gamma}^{1} \psi_{\varepsilon}(r) d r=0
$$

Date: December 23, 2015.

2000 Mathematics Subject Classification. 46E35; 42B15, 42B25.

Key words and phrases. Non-standard growth, variable exponent, Sobolev space, Gagliardo seminorm.

* Corresponding author. 
Examples of such families were given by Brézis [5, Remark 8].

We define

$$
\varrho_{\#}^{\varepsilon}(f):=\int_{0}^{1} \int_{\mathbb{R}^{n}}\left(\frac{1}{r} M_{B(x, r)}^{\#} f\right)^{p(x)} d x \psi_{\varepsilon}(r) d r
$$

where

$$
M_{B(x, r)}^{\#} f=f_{B(x, r)}\left|f(y)-f_{B(x, r)}\right| d y \quad \text { and } \quad f_{B(x, r)}=f_{B(x, r)} f(y) d y .
$$

For each function $\varrho_{\#}^{\varepsilon}$, we define the norm as usual from this by

$$
\|f\|_{\#}^{\varepsilon}:=\inf \left\{\lambda>0 \mid \varrho_{\#}^{\varepsilon}(f / \lambda) \leqslant 1\right\} .
$$

Our main result, Theorem 4.1, states that, for bounded exponents log-Hölder continuous, $p(\cdot)$, with ess $\inf _{x \in \mathbb{R}^{n}} p(x)>1$, an $L^{1}\left(\mathbb{R}^{n}\right)$ function $f$, has gradient in $L^{p(\cdot)}\left(\mathbb{R}^{n} ; \mathbb{R}^{n}\right)$ if and only if $\lim \sup _{\varepsilon \rightarrow 0^{+}} \varrho_{\#}^{\varepsilon}(f)<\infty$. Moreover, if $\nabla f \in L^{p(\cdot)}\left(\mathbb{R}^{n} ; \mathbb{R}^{n}\right)$, then its variable exponent modular as well as its $L^{p(\cdot)}$-norm can be obtained as limits of some functionals that do not involve the derivatives of $f$. Namely,

$$
\lim _{\varepsilon \rightarrow 0^{+}} \varrho_{\#}^{\varepsilon}(f)=\varrho_{p(\cdot)}\left(c_{n}|\nabla f|\right) \quad \text { and } \quad \lim _{\varepsilon \rightarrow 0^{+}}\|f\|_{\#}^{\varepsilon}=c_{n}\|\nabla f\|_{p(\cdot)}
$$

where

$$
c_{n}=f_{B(0,1)}\left|x \cdot e_{1}\right| d x .
$$

As mentioned above, the difference quotients functionals considered by Bourgain, Brézis, and Mironescu [3, 4], in the case of constant exponent, provide good imaging models. Also variable exponent variants of the ROF model have been proposed $[6,13,14]$. These models exhibit less staircasing than the classical models. It would be interesting to understand whether the functional defined through the sharp averaging operator $\varrho_{\#}^{\varepsilon}(f)$ can be applied to imaging problems when the natural growth depends on the region of the domain. Another open question is how fractional smoothness defined in this way relates to the variable exponent Besov and Triebel-Lizorkin spaces $[1,10]$.

\section{BACKGROUND MATERIAL}

We denote by $\mathbb{R}^{n}$ the $n$-dimensional real Euclidean space. We write $B(x, r)$ for the open ball in $\mathbb{R}^{n}$ centered at $x \in \mathbb{R}^{n}$ and radius $r>0$. We use $c$ as a generic positive constant, i.e. a constant whose value may change from appearance to appearance. If $E \subset \mathbb{R}^{n}$ is a measurable set, then $|E|$ stands for its (Lebesgue) measure and $\chi_{E}$ denotes its characteristic function.

We will need the following inequality

$$
(a+b)^{p} \leqslant(1+\varepsilon)^{p-1} a^{p}+\left(\frac{1+\varepsilon}{\varepsilon}\right)^{p-1} b^{p},
$$

valid for $p \geqslant 1$ and $\varepsilon>0$ and which follows from the weighted power-mean inequality

$$
\frac{a+\varepsilon \frac{b}{\varepsilon}}{1+\varepsilon} \leqslant \sqrt[p]{\frac{a^{p}+\varepsilon\left(\frac{b}{\varepsilon}\right)^{p}}{1+\varepsilon} .}
$$

We denote by $\mathcal{P}_{+}\left(\mathbb{R}^{n}\right)$ the set of all bounded measurable functions $p: \mathbb{R}^{n} \rightarrow[1, \infty)$, so-called variable exponents. For $A \subset \mathbb{R}^{n}$ and $p \in \mathcal{P}_{+}\left(\mathbb{R}^{n}\right)$ we denote $p_{A}^{+}=\operatorname{ess}_{\sup _{x \in A}} p(x)$ and $p_{A}^{-}=\operatorname{essinf}_{x \in A} p(x)$; we abbreviate $p^{+}=p_{\mathbb{R}^{n}}^{+}$and $p^{-}=p_{\mathbb{R}^{n}}^{-}$. 

if

Let $U \subset \mathbb{R}^{n}$. We say that $g: U \rightarrow \mathbb{R}$ satisfies the local log-Hölder continuity condition

for all $x, y \in U$. If

$$
|g(x)-g(y)| \leqslant \frac{c}{\log (e+1 /|x-y|)}
$$

$$
\left|g(x)-g_{\infty}\right| \leqslant \frac{c^{\prime}}{\log (e+|x|)}
$$

for some $g_{\infty} \geqslant 1, c^{\prime}>0$ and all $x \in U$, then we say $g$ satisfies the log-Hölder decay condition (at infinity). If both conditions are satisfied, we simply speak of log-Hölder continuity. By the log-Hölder constant we mean $\max \left\{c, c^{\prime}\right\}$. The class of bounded logHölder continuous exponents is denoted by $\mathcal{P}_{+}^{\log }\left(\mathbb{R}^{n}\right)$. In our analysis we will consider exponents in $\mathcal{P}_{+}^{\log }\left(\mathbb{R}^{n}\right)$ with $p^{-}>1$.

The variable exponent modular is defined by

$$
\varrho_{p(\cdot)}(f):=\int_{\mathbb{R}^{n}}|f(x)|^{p(x)} d x .
$$

The variable exponent Lebesgue space $L^{p(\cdot)}\left(\mathbb{R}^{n}\right)$ is the class of all measurable functions $f$ on $\mathbb{R}^{n}$ such that $\varrho_{p(\cdot)}(\lambda f)<\infty$ for some $\lambda>0$. This is a Banach space when equipped with the norm

$$
\|f\|_{p(\cdot)}:=\inf \left\{\lambda>0: \varrho_{p(\cdot)}(f / \lambda) \leqslant 1\right\} .
$$

If $p(x) \equiv p$ is constant, then $L^{p(\cdot)}=L^{p}$ is the classical Lebesgue space. Variable exponent Sobolev spaces are defined in an analogous way. For a detailed study of these spaces see the monographs $[9,11]$.

In the sequel we will often need to estimate integral functionals raised to some variable exponent introducing the exponent in the integrand. The following result is a variant of $\left[9\right.$, Theorem 4.2.4]: we do not need to assume $\|f\|_{p(\cdot)} \leqslant 1$ as in the original on account of taking a minimum of two terms on the left-hand side.

Lemma 2.2. Suppose $p \in \mathcal{P}_{+}^{\log }\left(\mathbb{R}^{n}\right), x \in \mathbb{R}^{n}$ and $\alpha, m>0$. Then there exists $c>0$ such that

$$
\min \left\{f_{B(x, r)}|f(y)| d y, r^{-\alpha}\right\}^{p(x)} \leqslant c f_{B(x, r)}|f(y)|^{p(y)} d y+c(e+|x|)^{-m},
$$

for every $f \in L_{\mathrm{loc}}^{p(\cdot)}\left(\mathbb{R}^{n}\right)$ and $0<r \leqslant 1$.

Proof. Denote $B:=B(x, r)$. By log-Hölder continuity,

$$
\min \left\{f_{B}|f(y)| d y, r^{-\alpha}\right\}^{p(x)-p_{B}^{-}} \leqslant r^{-\left(p(x)-p_{B}^{-}\right) \alpha} \leqslant c .
$$

Thus by Jensen's inequality,

$$
\min \left\{f_{B}|f(y)| d y, r^{-\alpha}\right\}^{p(x)} \leqslant c \min \left\{f_{B}|f(y)| d y, r^{-\alpha}\right\}^{p_{B}^{-}} \leqslant c f_{B}|f(y)|^{p_{B}^{-}} d y .
$$

Let $\frac{1}{s}=\frac{1}{p_{B}^{-}}-\frac{1}{p}$. By [9, Proposition 4.1.8], for every $m>0$, there exists $\gamma \in(0,1)$ such that $\gamma^{s(y)} \leqslant(e+|y|)^{-m}$, for all $y \in B$. Then, by Young's inequality [9, Lemma 3.2.15],

$$
\left(\frac{1}{\gamma}|f| \cdot \gamma\right)^{p_{B}^{-}} \leqslant\left(\frac{1}{\gamma}|f|\right)^{p(y)}+\gamma^{s(y)}
$$

from which the desired estimate follows. 


\section{Auxiliary results}

Let us start by proving our claim for regular functions. This result will be used in the final proof.

Lemma 3.1. Suppose that $p \in \mathcal{P}_{+}\left(\mathbb{R}^{n}\right)$. If $f \in C_{0}^{2}\left(\mathbb{R}^{n}\right)$, then

$$
\lim _{\varepsilon \rightarrow 0^{+}} \varrho_{\#}^{\varepsilon}(f)=\varrho_{p(\cdot)}\left(c_{n}|\nabla f|\right)
$$

where $c_{n}=f_{B(0,1)}\left|x \cdot e_{1}\right| d x$.

Proof. If $f \in C_{0}^{2}\left(\mathbb{R}^{n}\right)$, then a Taylor expansion gives

$$
f(y)=f(x)+\nabla f(x) \cdot(y-x)+R(x, y),
$$

where $R(x, y)=o(|x-y|)$ as $y \rightarrow x$. Hence

$$
\begin{aligned}
f(y)-f_{B(x, r)} & =f_{B(x, r)} f(y)-f(z) d z \\
& =f_{B(x, r)} \nabla f(x) \cdot(y-x)+R(x, y)-\nabla f(x) \cdot(z-x)-R(x, z) d z .
\end{aligned}
$$

By symmetry,

Thus we obtain

$$
f_{B(x, r)} \nabla f(x) \cdot(z-x) d z=0
$$

$$
f(y)-f_{B(x, r)}=\nabla f(x) \cdot(y-x)+R(x, y)-f_{B(x, r)} R(x, z) d z .
$$

Therefore, by the triangle inequality,

$$
\begin{aligned}
M_{B(x, r)}^{\#} f & =f_{B(x, r)}\left|\nabla f(x) \cdot(y-x)+R(x, y)-f_{B(x, r)} R(x, z) d z\right| d y \\
& \leqslant f_{B(x, r)}|\nabla f(x) \cdot(y-x)| d y+2 f_{B(x, r)}|R(x, y)| d y \\
& =|\nabla f(x)| f_{B(x, r)}\left|\frac{\nabla f(x)}{|\nabla f(x)|} \cdot(y-x)\right| d y+2 f_{B(x, r)}|R(x, y)| d y .
\end{aligned}
$$

Noting that $f_{B(x, r)}|\nu \cdot(y-x)| d y$ is independent of $\nu \in \mathcal{S}^{n-1}$, we have

$$
M_{B(x, r)}^{\#} f \leqslant c_{n} r|\nabla f(x)|+2 f_{B(x, r)}|R(x, y)| d y, \quad \text { where } \quad c_{n}:=f_{B(0,1)}\left|x \cdot e_{1}\right| d x .
$$

Similarly we derive the inequality

$$
M_{B(x, r)}^{\#} f \geqslant c_{n} r|\nabla f(x)|-2 f_{B(x, r)}|R(x, y)| d y .
$$

Note the lower bound $M_{B(x, r)}^{\#} f \geqslant 0$ also holds.

Defining $\Omega:=\left\{x \in \mathbb{R}^{n}:\left.f\right|_{B(x, 1)} \not \equiv 0\right\}$, we can write

$$
\varrho_{\#}^{\varepsilon}(f)=\int_{0}^{1} \int_{\Omega}\left(\frac{1}{r} M_{B(x, r)}^{\#} f\right)^{p(x)} d x \psi_{\varepsilon}(r) d r,
$$

since $M_{B(x, r)}^{\#} f=0$ when $x \notin \Omega$. Notice that $\Omega$ is bounded, since $f$ has compact support. 
From these estimates of $M_{B(x, r)}^{\#} f$, it follows that

$$
\begin{aligned}
\int_{0}^{1} \int_{\Omega} \max \left\{0, c_{n}|\nabla f(x)|-h(x, r)\right\}^{p(x)} d x \psi_{\varepsilon}(r) d r \\
\leqslant \varrho_{\#}^{\varepsilon}(f) \leqslant \int_{0}^{1} \int_{\Omega}\left(c_{n}|\nabla f(x)|+h(x, r)\right)^{p(x)} d x \psi_{\varepsilon}(r) d r
\end{aligned}
$$

where $h(x, r)=\frac{2}{r} f_{B(x, r)}|R(x, y)| d y$.

Let us start with the upper bound of $\varrho_{\#}^{\varepsilon}(f)$. For $\delta>0$ and bounded $p$ we have by $(2.1)$ that

$$
\left(c_{n}|\nabla f(x)|+h(x, r)\right)^{p(x)} \leqslant(1+\delta)^{p^{+}}\left(c_{n}|\nabla f(x)|\right)^{p(x)}+\left(\frac{1+\delta}{\delta}\right)^{p^{+}} h(x, r)^{p(x)} .
$$

Therefore, using (1.1) and (3.2), we find that

$$
\varrho_{\#}^{\varepsilon}(f) \leqslant(1+\delta)^{p^{+}} \int_{\Omega}\left(c_{n}|\nabla f(x)|\right)^{p(x)} d x+\left(\frac{1+\delta}{\delta}\right)^{p^{+}} \int_{0}^{1} \int_{\Omega} h(x, r)^{p(x)} d x \psi_{\varepsilon}(r) d r,
$$

and passing to the limit as $\varepsilon \rightarrow 0$ we get

$$
\begin{aligned}
\limsup _{\varepsilon \rightarrow 0} \varrho_{\#}^{\varepsilon}(f) \leqslant & (1+\delta)^{p^{+}} \varrho_{p(\cdot)}\left(c_{n}|\nabla f|\right)+ \\
& +\limsup _{\varepsilon \rightarrow 0}\left(\frac{1+\delta}{\delta}\right)^{p^{+}} \int_{\Omega} \int_{0}^{1} h(x, r)^{p(x)} \psi_{\varepsilon}(r) d r d x .
\end{aligned}
$$

To achieve the desired upper bound it remains to show that the limit on the right hand side is zero and then let $\delta \rightarrow 0$.

Let $\gamma>0$ be such that $\frac{|R(x, y)|}{|x-y|}<\varepsilon$ when $|x-y|<\gamma$. Since $f \in C_{0}^{2}\left(\mathbb{R}^{n}\right), \frac{R(x, y)}{|x-y|}$ is bounded. Thus

$$
h(x, r)=\frac{2}{r} f_{B(x, r)}|R(x, y)| d y \leqslant 2 f_{B(x, r)} \frac{|R(x, y)|}{|x-y|} d y \leqslant 2 \varepsilon \chi_{(0, \gamma)}(r)+c \chi_{(\gamma, 1)}(r) .
$$

Therefore, the defining property of $\psi_{\varepsilon}$ implies that

$$
\begin{aligned}
\int_{\Omega} \int_{0}^{1} h(x, r)^{p(x)} \psi_{\varepsilon}(r) d r d x & \leqslant \int_{\Omega}\left(\int_{0}^{\gamma}(2 \varepsilon)^{p(x)} \psi_{\varepsilon}(r) d r+c \int_{\gamma}^{1} \psi_{\varepsilon}(r) d r\right) d x \\
& \leqslant(2 \varepsilon)^{p^{-}}|\Omega|+c|\Omega| \int_{\gamma}^{1} \psi_{\varepsilon}(r) d r
\end{aligned}
$$

It is then clear, by (1.2), that the limit on the right hand side of (3.3) is zero, which finishes the proof of the upper bound.

To achieve the lower bound, observe that, using again (2.1), we get, for $\delta>0$,

$$
\max \left\{0, c_{n}|\nabla f(x)|-h(x, r)\right\}^{p(x)} \geqslant \frac{1}{(1+\delta)^{p^{+}}}\left(c_{n}|\nabla f(x)|\right)^{p(x)}-\frac{1}{\delta^{p^{+}}} h(x, r)^{p(x)} ;
$$

indeed the lower bound is trivial if the maximum equals zero. Therefore,

$$
\liminf _{\varepsilon \rightarrow 0} \varrho_{\#}^{\varepsilon}(f) \geqslant \frac{1}{(1+\delta)^{p^{+}}} \varrho_{p(\cdot)}\left(c_{n}|\nabla f|\right)-\limsup _{\varepsilon \rightarrow 0} \frac{1}{\delta^{p^{+}}} \int_{\Omega} \int_{0}^{1} h(x, r)^{p(x)} d x \psi_{\varepsilon}(r) d r .
$$

As already seen, this last limit is zero. The conclusion follows as before, by letting $\delta \rightarrow 0$. All in all, this gives $\lim _{\varepsilon \rightarrow 0} \varrho_{\#}^{\varepsilon}(f)=\varrho_{p(\cdot)}\left(c_{n}|\nabla f|\right)$, as was to be shown. 
Next we derive an auxiliary upper bound which holds for all functions in the homogeneous Sobolev space $D^{1, p(\cdot)}\left(\mathbb{R}^{n}\right)$ which consists of functions $u \in L_{\text {loc }}^{1}\left(\mathbb{R}^{n}\right)$ with $|\nabla u| \in$ $L^{p(\cdot)}\left(\mathbb{R}^{n}\right)$.

Lemma 3.4. Suppose that $p \in \mathcal{P}_{+}^{\log }\left(\mathbb{R}^{n}\right)$. If $u \in D^{1, p(\cdot)}\left(\mathbb{R}^{n}\right)$, then

$$
\varrho_{\#}^{\varepsilon}(u) \leqslant c \max \left\{\|\nabla u\|_{p(\cdot)}^{p^{-}},\|\nabla u\|_{p(\cdot)}^{p^{+}}\right\} .
$$

Proof. Let $u \in D^{1, p(\cdot)}\left(\mathbb{R}^{n}\right)$ be such that $\|\nabla u\|_{p(\cdot)} \leqslant 1$. By the Poincaré inequality,

$$
M_{B(x, r)}^{\#} u=f_{B(x, r)}\left|u(y)-u_{B(x, r)}\right| d y \leqslant c r f_{B(x, r)}|\nabla u(y)| d y .
$$

Applying this and Lemma 2.2, with $f=|\nabla u|$ and $m>n$, we obtain that

$$
\begin{aligned}
\varrho_{\#}^{\varepsilon}(u) & \leqslant c \int_{0}^{1} \int_{\mathbb{R}^{n}}\left(f_{B(x, r)}|\nabla u(y)| d y\right)^{p(x)} d x \psi_{\varepsilon}(r) d r \\
& \leqslant c \int_{0}^{1} \int_{\mathbb{R}^{n}} f_{B(x, r)}|\nabla u(y)|^{p(y)} d y+(e+|x|)^{-m} d x \psi_{\varepsilon}(r) d r .
\end{aligned}
$$

For the first integral we change the order of integration:

$$
\int_{\mathbb{R}^{n}} f_{B(x, r)}|\nabla u(y)|^{p(y)} d y d x=\int_{\mathbb{R}^{n}} \int_{\mathbb{R}^{n}}|\nabla u(y)|^{p(y)} \frac{\chi_{B(y, r)}(x)}{|B(x, r)|} d x d y=\int_{\mathbb{R}^{n}}|\nabla u(y)|^{p(y)} d y .
$$

Further we observe that $\int_{\mathbb{R}^{n}}(e+|x|)^{-m} d x<\infty$, since $m>n$. As $\int_{0}^{1} \psi_{\varepsilon}(r) d r=1$, we obtain that $\varrho_{\#}^{\varepsilon}(u) \leqslant c\left(\varrho_{p(\cdot)}(\nabla u)+1\right) \leqslant 2 c$.

Moreover, if we have a general, non-constant Sobolev function $u \in D^{1, p(\cdot)}\left(\mathbb{R}^{n}\right)$, the scaled version $u /\|\nabla u\|_{p(\cdot)}$ satisfies

$$
\varrho_{\#}^{\varepsilon}\left(\frac{u}{\|\nabla u\|_{p(\cdot)}}\right) \leqslant c\left(\varrho_{p(\cdot)}\left(\frac{\nabla u}{\|\nabla u\|_{p(\cdot)}}\right)+1\right)=2 c .
$$

It follows from the definition of $\varrho_{\#}^{\varepsilon}$ that $\varrho_{\#}^{\varepsilon}(u) \leqslant \varrho_{\#}^{\varepsilon}\left(\frac{u}{\lambda}\right) \max \left\{\lambda^{p^{-}}, \lambda^{p^{+}}\right\}$. Applying this with $\lambda=\|\nabla u\|_{p(\cdot)}$ in the previous inequality gives the claim.

\section{Main Results}

In this section we establish and prove our main result, which provides, in particular a characterization of variable exponent Sobolev spaces.

Theorem 4.1. Let $p \in \mathcal{P}_{+}^{\log }\left(\mathbb{R}^{n}\right)$ with $p^{-}>1$ and let $\left(\psi_{\varepsilon}\right)_{\varepsilon}$ be a family of functions satisfying (1.1) and (1.2). Assume $f \in L^{1}\left(\mathbb{R}^{n}\right)$. Then $|\nabla f| \in L^{p(\cdot)}\left(\mathbb{R}^{n}\right)$ if and only if

$$
\limsup _{\varepsilon \rightarrow 0^{+}} \varrho_{\#}^{\varepsilon}(f)<\infty \text {. }
$$

In this case,

$$
\lim _{\varepsilon \rightarrow 0^{+}} \varrho_{\#}^{\varepsilon}(f)=\varrho_{p(\cdot)}\left(c_{n}|\nabla f|\right)
$$

and

$$
\lim _{\varepsilon \rightarrow 0^{+}}\|f\|_{\#}^{\varepsilon}=c_{n}\|\nabla f\|_{p(\cdot)}
$$

where $c_{n}=f_{B(0,1)}\left|x \cdot e_{1}\right| d x$. 
Remark 4.5. We believe the result still holds under the weaker assumption $f \in L_{\text {loc }}^{1}\left(\mathbb{R}^{n}\right)$.

If $p^{-}=1$, then the previous theorem still holds provided the condition $\nabla f \in L^{p(\cdot)}\left(\mathbb{R}^{n}\right)$ is replaced by a suitable BV-variant, see [12], in particular [12, Proposition 6.3] for the compactness argument needed in part 2 of the proof.

Proof of Theorem 4.1. We divide the proof into three parts corresponding to the sufficient and necessary conditions for (4.2) and the equivalence of (4.3) and (4.4).

Part 1. We prove that (4.3) holds when $|\nabla f| \in L^{p(\cdot)}\left(\mathbb{R}^{n}\right)$, from which (4.2) follows, since then $\varrho_{p(\cdot)}\left(c_{n}|\nabla f|\right)<\infty$ as $p$ is bounded.

By the triangle inequality, for any $f, g \in L_{\text {loc }}^{1}\left(\mathbb{R}^{n}\right)$,

$$
\begin{aligned}
& f_{B(x, r)}\left|f(y)-f_{B(x, r)}\right| d y \\
& \quad \leqslant f_{B(x, r)}\left|f(y)-g(y)-(f-g)_{B(x, r)}+g(y)-g_{B(x, r)}\right| d y \\
& \quad \leqslant f_{B(x, r)}\left|f(y)-g(y)-(f-g)_{B(x, r)}\right| d y+f_{B(x, r)}\left|g(y)-g_{B(x, r)}\right| d y .
\end{aligned}
$$

This reads $M_{B(x, r)}^{\#} f \leqslant M_{B(x, r)}^{\#}(f-g)+M_{B(x, r)}^{\#} g$. On the other hand, by $(2.1)$ and since $p$ is bounded, $(a+b)^{p(x)} \leqslant(1+\delta)^{p^{+}} a^{p(x)}+c_{\delta} b^{p(x)}$. Combining these, we conclude that

$$
\varrho_{\#}^{\varepsilon}(f) \leqslant c_{\delta} \varrho_{\#}^{\varepsilon}(f-g)+(1+\delta)^{p^{+}} \varrho_{\#}^{\varepsilon}(g) \text {. }
$$

Let $f \in D^{1, p(\cdot)}\left(\mathbb{R}^{n}\right)$ and $g \in C_{0}^{\infty}\left(\mathbb{R}^{n}\right)$. By Lemmas 3.4 and 3.1,

$$
\limsup _{\varepsilon \rightarrow 0^{+}} \varrho_{\#}^{\varepsilon}(f) \leqslant c_{\delta} \max \left\{\|\nabla(f-g)\|_{p(\cdot)}^{p^{-}},\|\nabla(f-g)\|_{p(\cdot)}^{p^{+}}\right\}+(1+\delta)^{p^{+}} \varrho_{p(\cdot)}\left(c_{n}|\nabla g|\right) \text {. }
$$

In place of $g$, consider $g_{\nu} \in C_{0}^{\infty}\left(\mathbb{R}^{n}\right)$ such that $\nabla g_{\nu}$ converges to $\nabla f$ in $L^{p(\cdot)}\left(\mathbb{R}^{n}\right)$, as $\nu \rightarrow \infty$ [9, Proposition 12.2.7]. Then, letting $\nu \rightarrow \infty$, we obtain that

$$
\limsup _{\varepsilon \rightarrow 0^{+}} \varrho_{\#}^{\varepsilon}(f) \leqslant \lim _{\nu \rightarrow \infty}(1+\delta)^{p^{+}} \varrho_{p(\cdot)}\left(c_{n}\left|\nabla g_{\nu}\right|\right)=(1+\delta)^{p^{+}} \varrho_{p(\cdot)}\left(c_{n}|\nabla f|\right)
$$

since modular convergence follows from norm convergence [9, Lemma 2.1.9]. Finally we let $\delta \rightarrow 0$ and obtain the desired upper bound. The lower bound is proved similarly, with the estimate

$$
\varrho_{\#}^{\varepsilon}(f) \geqslant \frac{1}{(1+\delta)^{p^{+}}} \varrho_{\#}^{\varepsilon}\left(g_{\nu}\right)-\frac{c_{\delta}}{(1+\delta)^{p^{+}}} \varrho_{\#}^{\varepsilon}\left(g_{\nu}-f\right) .
$$

Part 2. We prove that if (4.2) holds, then $|\nabla f| \in L^{p(\cdot)}\left(\mathbb{R}^{n}\right)$.

Let $G$ be a standard mollifier, that is $G \in C_{0}^{\infty}(B(0,1))$ is positive and radially symmetric with $\int_{B(0,1)} G(x) d x=1$. Let $K>0$ be a constant such that $G \leqslant K_{\frac{\chi_{B(0,1)}}{|B(0,1)|}}$ and let us denote $H:=\frac{\chi_{B(0,1)}}{|B(0,1)|}$. Let $G_{\delta}(x)=\delta^{-n} G(x / \delta)$ and $H_{\delta}(x)=\delta^{-n} H(x / \delta)$. Then by the triangle inequality and a change of integration order we find that,

$$
\begin{aligned}
M_{B(x, r)}^{\#}\left(G_{\delta} * f\right) & =f_{B(x, r)}\left|\left(G_{\delta} * f\right)(y)-\left(G_{\delta} * f\right)_{B(x, r)}\right| d y \\
& =f_{B(x, r)}\left|\left(G_{\delta} *\left(f-f_{B(\cdot, r)}\right)\right)(y)\right| d y \leqslant\left(G_{\delta} * M_{B(\cdot, r)}^{\#} f\right)(x) .
\end{aligned}
$$


On the other hand, by the Poincaré inequality,

$$
\begin{aligned}
\frac{1}{r} M_{B(x, r)}^{\#}\left(G_{\delta} * f\right) & \leqslant c f_{B(x, r)}\left|\nabla\left(G_{\delta} * f\right)(y)\right| d y=\frac{c}{\delta} f_{B(x, r)}\left|(\nabla G)_{\delta} * f(y)\right| d y \\
& \leqslant c \delta^{-1}\left\|(\nabla G)_{\delta}\right\|_{\infty}\|f\|_{L^{1}} \leqslant c \delta^{-n-1}
\end{aligned}
$$

Denote $g_{r}(x):=\frac{1}{r} M_{B(x, r)}^{\#} f$ and observe that $G_{\delta} * g_{r} \leqslant K H_{\delta} * g_{r}$. The previous two inequalities can be summarized as

$$
\frac{1}{r} M_{B(x, r)}^{\#}\left(G_{\delta} * f\right) \leqslant c \min \left\{H_{\delta} * g_{r}(x), \delta^{-n-1}\right\} .
$$

By Lemma 2.2, noting that $H_{\delta} * g_{r}$ is the integral average of $g_{r}$, we obtain that

$$
\min \left\{H_{\delta} * g_{r}(x), \delta^{-n-1}\right\}^{p(x)} \leqslant c H_{\delta} *\left(g_{r}^{p(\cdot)}\right)(x)+c(e+|x|)^{-m} .
$$

Hence

$$
\int_{\mathbb{R}^{n}}\left(\frac{1}{r} M_{B(x, r)}^{\#}\left(G_{\delta} * f\right)\right)^{p(x)} d x \leqslant c \int_{\mathbb{R}^{n}} H_{\delta} *\left(g_{r}^{p(\cdot)}\right)(x) d x+c \int_{\mathbb{R}^{n}}(e+|x|)^{-m} d x .
$$

Here the second integral on the right hand side is bounded by a constant, if we choose $m>n$. For the first integral we change the order of integration:

$$
\int_{\mathbb{R}^{n}} \int_{\mathbb{R}^{n}} H_{\delta}(x-z) g_{r}(z)^{p(z)} d z d x=\int_{\mathbb{R}^{n}} \int_{\mathbb{R}^{n}} H_{\delta}(x-z) d x g_{r}(z)^{p(z)} d z=\int_{\mathbb{R}^{n}} g_{r}(z)^{p(z)} d z .
$$

Since $g_{r}(x)=\frac{1}{r} M_{B(x, r)}^{\#} f$, we conclude that

$$
\int_{\mathbb{R}^{n}}\left(\frac{1}{r} M_{B(x, r)}^{\#}\left(G_{\delta} * f\right)\right)^{p(x)} d x \leqslant c \int_{\mathbb{R}^{n}}\left(\frac{1}{r} M_{B(x, r)}^{\#} f\right)^{p(x)} d x+c .
$$

Multiplying by $\psi_{\varepsilon}(r)$ and integrating over $r \in[0,1]$, we find that

$$
\varrho_{\#}^{\varepsilon}\left(G_{\delta} * f\right) \leqslant c \varrho_{\#}^{\varepsilon}(f)+c .
$$

Suppose then that $\lim _{\varepsilon \rightarrow 0^{+}} \varrho_{\#}^{\varepsilon}(f)<\infty$ and note that $G_{\delta} * f \in C^{2}\left(\mathbb{R}^{n}\right)$. By Lemma 3.1 (in a bounded domain instead of a compactly supported function),

$$
\int_{B(0, R)}\left|\nabla\left(G_{\delta} * f\right)\right|^{p(x)} d x \leqslant \lim _{\varepsilon \rightarrow 0} \varrho_{\#}^{\varepsilon}\left(G_{\delta} * f ; B(0, R+\delta)\right) \leqslant c \lim _{\varepsilon \rightarrow 0} \varrho_{\#}^{\varepsilon}(f)+c=c .
$$

Here the upper bound is independent of $R$. Therefore $\nabla\left(G_{\delta} * f\right)$ is a bounded sequence in $L^{p(\cdot)}\left(\mathbb{R}^{n}\right)$. Since $1<p^{-} \leqslant p^{+}<\infty$, a subsequence weakly converges in $L^{p(\cdot)}\left(\mathbb{R}^{n}\right)$ to a function $l \in L^{p(\cdot)}\left(\mathbb{R}^{n}\right)[9$, Theorem 3.4.7]. It follows by the definition of the derivative that $l=\nabla f$, since $G_{\delta} * f$ converges to $f$ in $L_{\text {loc }}^{1}\left(\mathbb{R}^{n}\right)$. Thus $|\nabla f| \in L^{p(\cdot)}\left(\mathbb{R}^{n}\right)$.

Part 3. Finally we observe that we obtain (4.4) from (4.3) as follows: let $\delta>0$ and set $g:=f /\left(c_{n}(1+\delta)\left(\|\nabla f\|_{p(\cdot)}+\delta\right)\right)$. By (4.3) for $g$ it follows that

$$
\begin{aligned}
\lim _{\varepsilon \rightarrow 0} \varrho_{\#}^{\varepsilon}(g)=\varrho_{p(\cdot)}\left(c_{n}|\nabla g|\right)=\varrho_{p(\cdot)}\left(\frac{|\nabla f|}{(1+\delta)\left(\|\nabla f\|_{p(\cdot)}+\delta\right)}\right) & \leqslant \frac{1}{1+\delta} \varrho_{p(\cdot)}\left(\frac{|\nabla f|}{\|\nabla f\|_{p(\cdot)}+\delta}\right) \\
& \leqslant \frac{1}{1+\delta} .
\end{aligned}
$$

Hence for all sufficiently small $\varepsilon, \varrho_{\#}^{\varepsilon}(g) \leqslant 1$. Thus also $\|g\|_{\#}^{\varepsilon} \leqslant 1$, and so $\|f\|_{\#}^{\varepsilon} \leqslant$ $c_{n}(1+\delta)\left(\|\nabla f\|_{p(\cdot)}+\delta\right)$. Then we let $\delta \rightarrow 0$ to obtain one inequality of (4.4). The other is obtained analogously. 


\section{Connection With Difference Quotient Formulation}

In this section we consider how the constant exponent formulation in terms of difference quotients compares to ours. The classical formulation is based on the family of functionals

$$
D Q_{\varepsilon}(f):=\int_{\mathbb{R}^{n}} \int_{\mathbb{R}^{n}}\left(\frac{|f(x)-f(y)|}{|x-y|}\right)^{p} \varphi_{\varepsilon}(|x-y|) d x d y,
$$

where $\varphi_{\varepsilon}(r):=\psi_{\varepsilon}(r) r^{1-n}$. In this classical setting, the results obtained by Bourgain, Brézis, and Mironescu in [3,4] assert, in particular, that, for $p>1$, an $L^{p}$ function $f$ is in $W^{1, p}\left(\mathbb{R}^{n}\right)$ if and only if $\limsup _{\varepsilon \rightarrow 0} D Q_{\varepsilon}(f)$ is finite, in which case the limit exists and can be expressed in terms of the $L^{p}$-norm of $\nabla f$.

Here we will show that, for any $f \in D^{1, p}\left(\mathbb{R}^{n}\right)$,

$$
\lim _{\varepsilon \rightarrow 0} D Q_{\varepsilon}(f)=\lim _{\varepsilon \rightarrow 0} \int_{\mathbb{R}^{n}} \int_{0}^{1}\left(\frac{1}{r} M_{p, B(x, r)}^{\#} f\right)^{p} \xi_{\varepsilon}(r) d r d x
$$

where

$$
M_{p, B(x, r)}^{\#} f:=\left(f_{B(x, r)}\left|f(y)-f_{B(x, r)}\right|^{p} d y\right)^{\frac{1}{p}}
$$

and $\xi_{\varepsilon}$ is a function satisfying, up to a constant, (1.1) and (1.2).

Comparing this expression with (1.3), we see that the only difference is that the classical approach uses $M_{p, B(x, r)}^{\#} f$ whereas we used $M_{1, B(x, r)}^{\#} f$. A direct consequence of Jensen's inequality is that $M_{1, B(x, r)}^{\#} f \leqslant M_{p, B(x, r)}^{\#} f$ for $p \geqslant 1$. Hence the classical approach uses a marginally larger functional.

Next we prove our assertion. Suppose that $f \in D^{1, p}\left(\mathbb{R}^{n}\right)$ is fixed. Let $\left(\psi_{\varepsilon}\right)_{\varepsilon}$ be, as before, a family of functions satisfying (1.1) and (1.2), and assume also that $\psi_{\varepsilon}$ is extended to $\mathbb{R}^{+}$getting $\psi_{\varepsilon} \in C^{1}((0, \infty))$ with $\psi_{\varepsilon}(r)=0$ when $r \geqslant 1$. Let us denote

$$
F(x, r):=\int_{B(x, r)}|f(x)-f(y)|^{p} d y
$$

Note that

$$
F^{\prime}(x, r)=\int_{S(x, r)}|f(x)-f(y)|^{p} d y
$$

Then

$$
D Q_{\varepsilon}(f)=\int_{\mathbb{R}^{n}} \int_{0}^{1} F^{\prime}(x, r) r^{-p} \varphi_{\varepsilon}(r) d r d x
$$

since $\psi_{\varepsilon}(r)=0$ for $r \geqslant 1$. We abbreviate $r^{-p} \varphi_{\varepsilon}(r)=: \bar{\varphi}_{\varepsilon}(r)$. In the inner integral we use integration by parts:

$$
\int_{0}^{1} F^{\prime}(x, r) \bar{\varphi}_{\varepsilon}(r) d r=-\lim _{r \rightarrow 0} F(x, r) \bar{\varphi}_{\varepsilon}(r)-\int_{0}^{1} F(x, r) \bar{\varphi}_{\varepsilon}^{\prime}(r) d r,
$$

since $\varphi_{\varepsilon}(1)=0$. We show that the limit equals zero.

For this we estimate

$$
r^{-n} F(x, r) \leqslant c f_{B(x, r)}\left|f(y)-f_{B(x, r)}\right|^{p} d y+c\left|f(x)-f_{B(x, r)}\right|^{p} .
$$


The first term is bounded by $c r^{p} f_{B(x, r)}|\nabla f|^{p} d y$ (Poincaré's inequality) while the second is bounded by $\operatorname{cr}^{p}(M|\nabla f|)^{p}$ (Riesz' potential estimate, eg. [9, Lemmas 6.1.4 and 8.2.1]), where $M$ denotes the maximal operator. Hence

$$
r^{-n-p} F(x, r) \leqslant c f_{B(x, r)}|\nabla f(y)|^{p} d y+c M|\nabla f|(x)^{p} \leqslant c M|\nabla f|(x)^{p} .
$$

for almost every $x$, since the average integral can be estimated by the maximal function. Thus for almost every $x \in \mathbb{R}^{n}$,

$$
0 \leqslant \lim _{r \rightarrow 0} F(x, r) \bar{\varphi}_{\varepsilon}(r) \leqslant c M|\nabla f|(x)^{p} \lim _{r \rightarrow 0} r^{n} \varphi_{\varepsilon}(r)=0 .
$$

It follows that

$$
D Q_{\varepsilon}(f)=-\int_{\mathbb{R}^{n}} \int_{0}^{1} F(x, r) \bar{\varphi}_{\varepsilon}^{\prime}(r) d r d x=\int_{\mathbb{R}^{n}} \int_{0}^{1} f_{B(x, r)} \frac{|f(x)-f(y)|^{p}}{r^{p}} d y \xi_{\varepsilon}(r) d r d x,
$$

where

$$
\xi_{\varepsilon}(r):=-|B(0, r)| r^{p} \bar{\varphi}_{\varepsilon}^{\prime}(r)=c\left(p r^{n-1} \varphi_{\varepsilon}(r)-r^{n} \varphi_{\varepsilon}^{\prime}(r)\right) .
$$

We see that $\xi_{\varepsilon}$ satisfies conditions (1.1) and (1.2) up to a constant. In particular, if $\varphi_{\varepsilon}(r)=\frac{\varepsilon}{r^{n-\varepsilon}}$, then $\xi_{\varepsilon}=|B(0,1)|(p+n-\varepsilon) r^{n-1} \psi_{\varepsilon}$.

We every $\delta>0$, we have by (2.1) that

$$
|| f(x)-\left.f(y)\right|^{p}-\left|f(y)-f_{B(x, r)}\right|^{p}|\leqslant \delta| f(x)-\left.f(y)\right|^{p}+c_{\delta}\left|f(x)-f_{B(x, r)}\right|^{p} .
$$

With this estimate and the inequality $\left|f(x)-f_{B(x, r)}\right| \leqslant c r M|\nabla f|$ from above, we can show that, at the limit, $f(x)$ in the above formula for $D Q_{\varepsilon}$ can be changed to $f_{B(x, r)}$. In other words, we obtain that

$$
\lim _{\varepsilon \rightarrow 0} D Q_{\varepsilon}(f)=\lim _{\varepsilon \rightarrow 0} \int_{\mathbb{R}^{n}} \int_{0}^{1}\left(\frac{1}{r} M_{p, B(x, r)}^{\#} f\right)^{p} \xi_{\varepsilon}(r) d r d x .
$$

It would be possible to cover both the classical functional and the variable exponent version with the expression

$$
\lim _{\varepsilon \rightarrow 0} \int_{\mathbb{R}^{n}} \int_{0}^{1}\left(\frac{1}{r} M_{p^{-}, B(x, r)}^{\#} f\right)^{p(x)} \xi_{\varepsilon}(r) d r d x
$$

where the exponent is "split" so that the global minimum is taken inside the sharp maximal function and the variable part is left outside.

\section{ACKNOWLEDGEMENTS}

This work was partially supported by the Fundação para a Ciência e a Tecnologia (Portuguese Foundation for Science and Technology) through the projects UID/MAT/00297/ 2013 (Centro de Matemática e Aplicações) and EXPL/MAT-CAL/0840/2013. Part of this work was done while the authors enjoyed the hospitality of University of Turku and of the Centro de Matemática e Aplicações (CMA), Faculdade de Ciências e Tecnologia, Universidade Nova de Lisboa.

We thank the referee for some useful observations. 


\section{REFERENCES}

[1] A. Almeida and P. Hästö: Besov spaces with variable smoothness and integrability, J. Funct. Anal. 258 (2010), no. 5, 1628-1655.

[2] G. Aubert and P. Kornprobst: Can the nonlocal characterization of Sobolev spaces by Bourgain et al. be useful for solving variational problems? SIAM J. Numer. Anal. 47, (2009), 844-860.

[3] J. Bourgain, H. Brézis, and P. Mironescu: Another look at Sobolev spaces, Optimal control and partial differential equations. In honour of Professor Alain Bensoussan's 60th birthday. Proceedings of the conference, Paris, France, December 4, 2000 (2001), 439-455.

[4] J. Bourgain, H. Brézis, and P. Mironescu: Limiting embedding theorems for $W^{s, p}$ when $s \uparrow 1$ and applications, J. Anal. Math. 87 (2002), 77-101.

[5] H. Brézis: How to recognize constant functions. Connections with Sobolev spaces, Russ. Math. Surv. 57 (2002), no. 4, 693-708.

[6] Y. Chen, S. Levine, and R. Rao: Variable exponent, linear growth functionals in image restoration, SIAM J. Appl. Math. 66 (2006), no. 4, 1383-1406.

[7] D. Cruz-Uribe and A. Fiorenza: Variable Lebesgue Spaces. Foundations and Harmonic Analysis, Birkhäuser/Springer, New York, 2013.

[8] L. Diening and P. Hästö: Variable exponent trace spaces, Studia Math. 183 (2007) 127-141.

[9] L. Diening, P. Harjulehto, P. Hästö, and M. Rưžička: Lebesgue and Sobolev Spaces with Variable Exponents, Lecture Notes in Mathematics, vol. 2017, Springer-Verlag, Berlin, 2011.

[10] L. Diening, P. Hästö and S. Roudenko: Function spaces of variable smoothness and integrability, $J$. Funct. Anal. 256 (2009), no. 6, 1731-1768.

[11] A. Fiorenza: A mean continuity type result for certain Sobolev spaces with variable exponent, Commun. Contemp. Math. 4 (2002), 587-605.

[12] P. Harjulehto, P. Hästö, and V. Latvala: Minimizers of the variable exponent, non-uniformly convex Dirichlet energy, J. Math. Pures Appl. (9) 89 (2008), no. 2, 174-197.

[13] P. Harjulehto, P. Hästö, V. Latvala, and O. Toivanen: Critical variable exponent functionals in image restoration, Appl. Math. Letters 26 (2013), 56-60.

[14] F. Li, Z. Li, and L. Pi: Variable exponent functionals in image restoration, Appl. Math. Comput. 216 (2010), no. 3, 870-882.

[15] L. Rudin, S. Osher, and E. Fatemi: Nonlinear total variation based noise removal algorithms, Physica D 60, (1992), no.1-4, 259-268.

Department of Mathematical Sciences, P.O. Box 3000, Fi-90014 University of Oulu, Finland, And, Department of Mathematics and Statistics, University of Turku, Finland E-mail address: peter.hasto@oulu.fi

$U R L:$ http://cc.oulu.fi/ phasto/

Centro de Matemática e Aplicações (CMA) and Departamento de Matemática, Faculdade de Ciências e Tecnologia, Universidade Nova de Lisboa, Quinta da Torre, 2829-516 Caparica, Portugal

E-mail address: amfr@fct.unl.pt

$U R L:$ https://sites.google.com/site/anaribeirowebpage/home 